\title{
Una nueva obra de Johannes de Lairesse: La Alegoría del Dibujo
}

\author{
A new painting by Johannes de Lairesse: The Allegory of Drawing
}

\author{
Matías Díaz Padrón ${ }^{1}$ \\ Académie Royale d'Archélogie et d'Histoire de l'Art de Belgique \\ Instituto Moll. Centro de investigación en pintura flamenca
}

\begin{abstract}
Resumen: En este trabajo se identifica a Johannes de Lairesse como autor de la Alegoría del Dibujo anteriormente catalogada a nombre de su padre, Gérard de Lairesse. La confusión entre el trabajo de ambos se debe, en parte, a que Jan sigue composiciones de su progenitor. La que aquí se estudia presenta una compleja iconografía que ha podido ser aclarada como la Alegoría del Dibujo, cuando antes estaba confundida con Aquiles descubierto entre las hijas de Licómedes.
\end{abstract}

Palabras Clave: Johannes de Lairesse, Gérard de Lairesse, Alegoría del Dibujo, siglo XVII, Rouen.

Abstract: In this paper, Johannes de Lairesse is identified as the author of an Allegory of Drawing which was previously catalogued as a work of his father, Gérard de Lairesse. The production of both has been misled, because the close connection with Jan's compositions following their father's. This paint has a complex iconography, which is here clarified and related to the Allegory of Drawing, but before was mistaken with Achilles recognised among the Daughters of Lycomedes.

Key Words: Johannes de Lairesse, Gérard de Lairesse, Allegory of Drawing, $17^{\text {th }}$ century, Rouen.



a pintura de Johannes de Lairesse (Ámsterdam, 16731748) con la Alegoría del Dibujo, de exquisita calidad y medianas dimensiones (L. $82 \times 94,5 \mathrm{~cm}$.) ${ }^{2}$, estuvo catalogada a nombre de su padre, Gérard de Lairesse (Lieja, 1641 - Ámsterdam, 1717), e identificada como la historia de Aquiles

${ }^{1}$ ORCID iD: http://orcid.org/0000-0002-5137-7583

2 En uno de los travesaños del bastidor constan el número 130 y una etiqueta con el nombre de "Hoffman".

(C) 2018 Philostrato. Revista de Historia y Arte 
descubierto entre las hijas de Licómedes, antes de pasar a la colección Epiarte $^{3}$. (Fig. 1) Es razonable la confusión entre los dos pintores, pues Johannes de Lairesse es fiel al estilo y a la técnica de su progenitor, que fue también su maestro. Por tanto, el enredo entre la producción de uno y otro pintor es habitual en la bibliografía, a pesar de las firmas ${ }^{4}$, y no sorprende la errónea atribución de esta obra que se estudia a Gérard cuando estuvo en el mercado artístico internacional. Sin embargo, el análisis más atento de la pintura y la localización de una réplica firmada por Johannes de Lairesse en el Museo de Bellas Artes de Rouen ${ }^{5}$, han permitido fijar la autoría del hijo y rectificar el tema representado ${ }^{6}$. (Fig. 2)

Poco se conoce de Johannes de Lairesse. Nacido en $1673^{7}$, fue el segundo hijo de Gérard de Lairesse, con quien se formó y trabajó, siguiendo fielmente su estilo, tanto en la técnica como en la composición y la temática. Es evidente en su obra la vuelta al clasicismo según las consignas que marcó Nicolas Poussin (Les Andelys, 1594-Roma, 1665), en contraposición a lo que Rubens imponía desde Flandes en el siglo XVII. Poco después de Rubens, otra corriente pictórica va a hacer prevalecer el equilibrio armónico de la composición, la disciplina y la belleza, frente al movimiento exacerbado y el frenético dinamismo de las composiciones. La añoranza a la Antigüedad clásica va a ser más evidente en esta nueva corriente, que va a imperar a fines del siglo XVII, tanto en Flandes como en Holanda ${ }^{8}$. Es una vuelta al clasicismo, no sólo en la forma sino también en el fondo. Esto se presentía en la generación de los discípulos de Rubens, en especial en la figura de Erasmus Quellinus II (1607-1678) ${ }^{9}$. La diagonal barroca va dejando sitio a la horizontal acompasada y al dibujo como fundamento principal en la pintura. Es lo que domina en la estética de Gérard de Lairesse y de su hijo, que le siguió con igual fortuna, justificando el equívoco de la catalogación de las obras de uno y otro.

\footnotetext{
3 Mónaco (Christie's), 2-XII-1989, nº15.

${ }^{4}$ Es el caso del Juicio de Salomón, que se atribuyó a Gérard de Lairesse en Nueva York (Christie's, 22-031978). También Alejandro y Roxana (Galería Lempertz,11-11-1955); Marte, Venus y Mercurio del Museo de Copenhague; Rinaldo y Armida, (Christie's. 29-05-1981); En el jardín de Armida (Sotheby's, 8-021978); Juicio de Salomón, (Christie's, 27-02-1981); Alegoría del vicio y la virtud, (Sotheby's, 19-041989); Antioques y Stratonice, (Galería Fabert, 31-05-1990); Alain Roy, Gerard de Lairesse 1640-1711, (Paris: Arthena, 1992), p. 165.

5 Diederik Bakhuÿs, Jasper Hillegers, Cécile Tainturier y Koenraad Jonckheers, Tableaux flamands et hollandais du Musée des Beaux-Arts de Rouen, (Paris: Fondation Custodia, 2009), pp. 31-124, 209.

${ }^{6}$ Eddy de Jongh, "The Artist's Apprentice and Minerva's Secret: An Allegory of Drawing by Jan de Lairesse", Simiolus: Netherlands Quarterly for the History of Art, 13, no 3/4, (1983), p. 207.

7 Ha habido bastantes dudas respecto al año de su nacimiento. Wurzbach lo supone hacia 1661; y Roy lo apunta hacia 1674. Alfred von Wurzbach, Niederländisches Künstler-Lexikon, II, (Wien-Leipzig: Halm und Goldmann, 1910, p. 9; Roy, Gérard de Lairesse, p. 132. Ecartico, en cambio, especifica su fecha de bautismo en Ámsterdam el 24 de septiembre de 1673 y su enterramiento en la misma ciudad el 11 de junio de 1748. (ECARTICO. Amsterdam Centre for the Study of the Golden Age. University of Amsterdam). En web: http://www.vondel.humanities.uva.nl/ecartico/persons/4441; (visitada: 5/12/2018)

8 En Flandes, Theodoor van Loon, va a ser uno de sus grandes abanderados. En los Países Bajos, en cambio, esta tendencia va a estar mucho más extendida entre Adriaen van der Werff, Jacob de Wit o el propio Gerard de Lairesse. Th. Cornil, "Théodore van Loon et la peinture italienne", Bulletin de I'Institut historique belge de Rome, 17, (1936), pp. 187-211; Exp., Theoodor van Loon. A Caravaggist painter between Rome and Brussels, BOZAR, Bruselas, 2018-2019; Albert Blankert et al., Hollands Classicisme in de zeventiende-eeuwse schilderkusnt, Cat. Exp., (Rotterdam-Frankfurt am Main, 1999), pp. 338-340.

9 Jean-Pierre de Bruyn, Erasmus II Quellinus (1607-1678) De Schilderijen met Catalogue Raisonné. Vlaamse Schilders uit de Tijd Van de Grote Meesters, (Freren: Luca, 1988).
} 


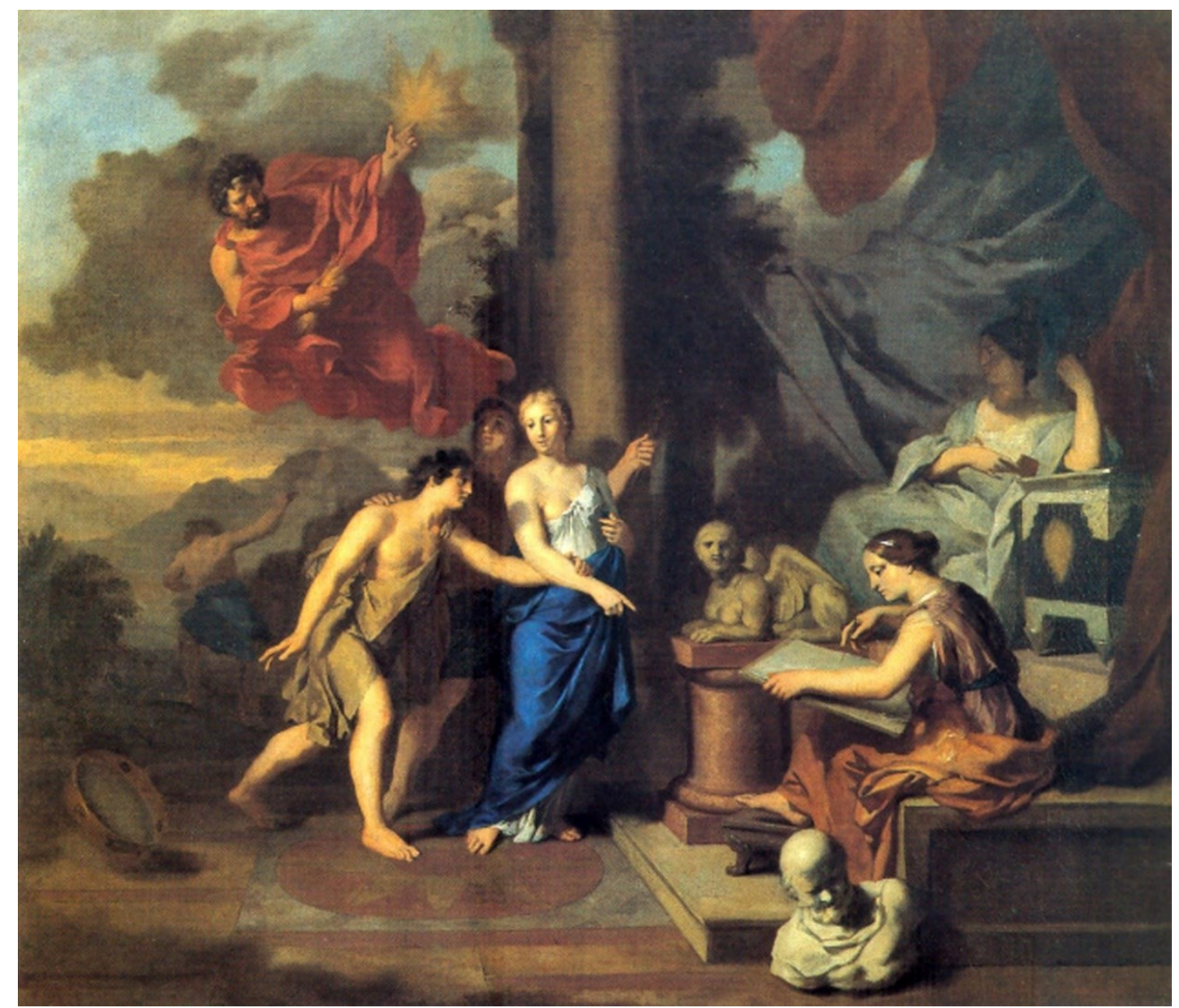

Fig. 1. Johannes de Lairesse, Alegoría del Dibujo. Colección Epiarte (inv. n 130)

El asunto de esta pintura se confundió con la historia de Aquiles descubierto entre las hijas de Licómedes $^{10}$, tema frecuente en la producción de Gérard de Lairesse, como prueban las repeticiones de los museos de Estocolmo, La Haya y Brunswick ${ }^{11}$. Lo que se representa en el lienzo es un tema muy distinto. Júpiter emerge desde lo alto, entre oscuros nubarrones de un dorado atardecer. Con su mano izquierda apunta a una estrella reluciente y porta un rayo en la derecha. Debajo Júpiter, aparecen otros dioses junto a la figura alegórica del dibujo, sentada en una plataforma de piedra con un ampuloso cortinaje que pende de lo alto. Una envolvente tonalidad armoniza el conjunto, con una columna afirmando el eje de la composición, como es habitual en la producción de Gérard de Lairesse. Esta columna y la distribución espacial la repite en su pintura Júpiter envía a Iris a Juno para ordenarle que devuelva el cuerpo de Héctor de la antigua colección del Barón

\footnotetext{
${ }^{10}$ Natale Conti, Mythologiae, sive explicationis fabularum. Libri Decem, (Paris: Thomas Blasius, 1605), pp. 530-556.

${ }^{11}$ Repeticiones del Nationalmuseum de Estocolmo (inv. NM 494), Mauritshuis de La Haya (inv. 82) y del Herzog Anton-Ulrich Museum de Brunswick (inv. 287). Roy, Gérard de Lairesse, pp. 236-237, $335-336$ y 267-268.
} 




Fig. 2. Jan de Lairesse, Alegoría del Dibujo. Museo de Bellas Artes de Rouen (inv. nº 975-4-80)

de Brienen (París) y en su grabado de Mercurio ordena a y Calipso dejar partir a Ulises ${ }^{12}$.

El mensaje de la Alegoría del dibujo es hermético y complejo. Es la reivindicación del dibujo como categoría fundamental en el arte de la pintura, frente al color que imperó en Rubens. La obra lo transmite a través de todo un abanico de signos y símbolos de compleja comprensión. Júpiter, con el rayo y la estrella, luz de la inspiración y del esfuerzo ${ }^{13}$, ilumina el alma del joven aprendiz que centra el discurso. Éste se apoya en el brazo de la mujer que se identifica con la Razón (Ratio), sujetando la brida y señalando hacia la personificación del Dibujo que le muestra al joven aprendiz ${ }^{14}$. Detrás está Mercurio, semioculto en la penumbra, con sus símbolos habituales: la capa, el sombrero y las sandalias aladas y el caduceo. Apoya la mano derecha sobre el hombro del joven protagonista de la narrativa, al tiempo que vuelve su mi-

\footnotetext{
12 Roy, Gerard de Lairesse, pp. 291-293 y 442-443.

13 Cesare Ripa, Iconologia of Uytbeeldingen des Verstands, (Amsterdam: Dirck Pietersz Pers, 1644), p. 472.; Vid. De Jongh, "Artist's Apprentice", p. 209.

14 De Jongh, "Artist's Apprentice", p. 203.
} 


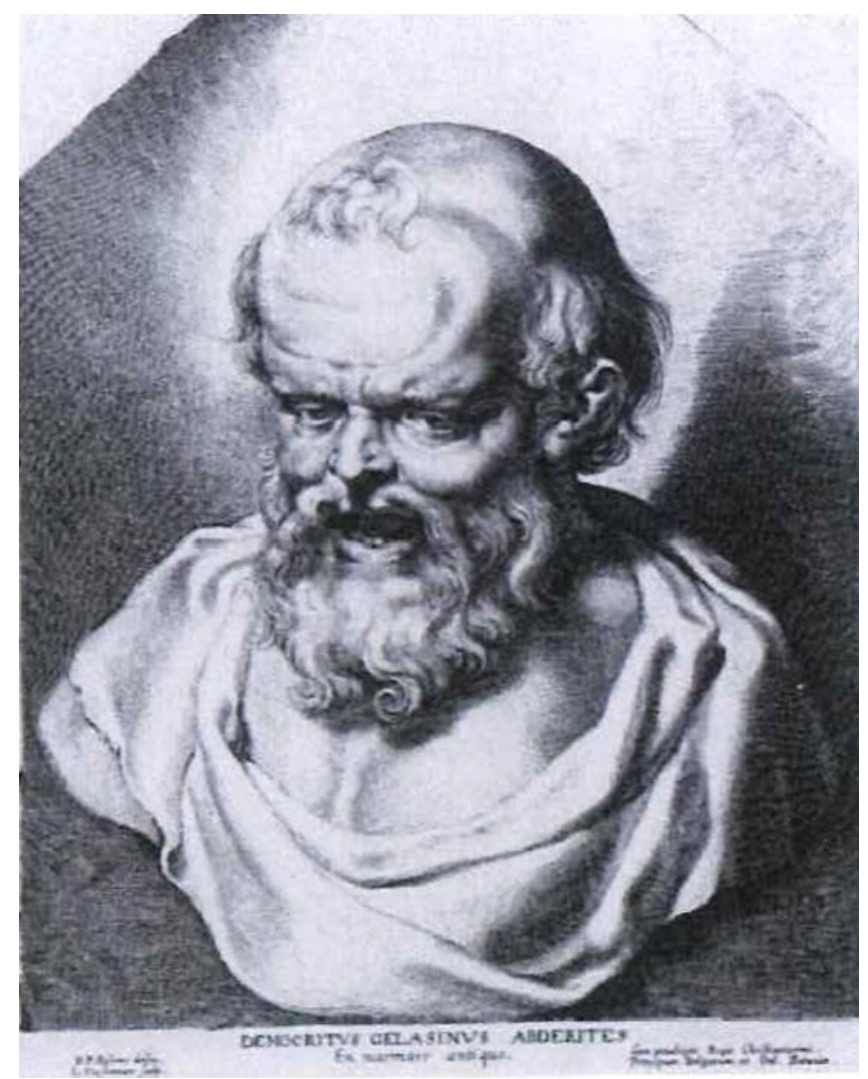

Fig. 3. Lucas Vorsterman siguiendo un dibujo de Rubens, Demócrito, grabado.

rada hacia Júpiter. El aprendiz se ha desprendido de la pandereta que está a sus espaldas, en tierra. Es un gesto de renuncia a la diversión a la que estuvo entregado en detrimento del trabajo. El Trabajo está simbolizado por Baco, que conduce unos bueyes en el paisaje del fondo, cuyo horizonte se abre a un dorado atardecer.

Junto a la Razón, Mercurio conduce al joven ante Minerva, quien encarna la Sabiduría, acomodada en un trono y sumergida en la penumbra de un amplio cortinaje en el lado derecho de la escena. La diosa reposa el brazo izquierdo en un arca de oro, a la vez que con la mano derecha toma una llave de gran tamaño, cuyo extremo está apoyado en el arca. La esfinge de piedra es fuente de inspiración y atributo de Minerva, no un simple complemento decorativo. Esta esfinge parece tomar vida al tener el rostro girado hacia la alegoría del Dibujo: la mujer que dibuja a su lado sentada en un podio. En el primer plano, sobre el suelo, destaca un busto de Demócrito en yeso, con el contenido moral de su pensamiento filosófico, copia del grabado de Lucas Vorsterman, según dibujo de Rubens ${ }^{15}$ (fig. 3).

Aquí, el joven se entrega a su vocación, ayudado por la Razón, Júpiter y Minerva. Al comparar la pintura con la réplica del museo Rouen, se ve que se ha prescindido de algunos atributos. Minerva ya no lleva la lanza que porta en su mano izquierda y la tablita del primer plano con dibujos geométricos

${ }^{15}$ Bakhuÿs et al., Tableaux flamands, p. 209, fig. 49. 


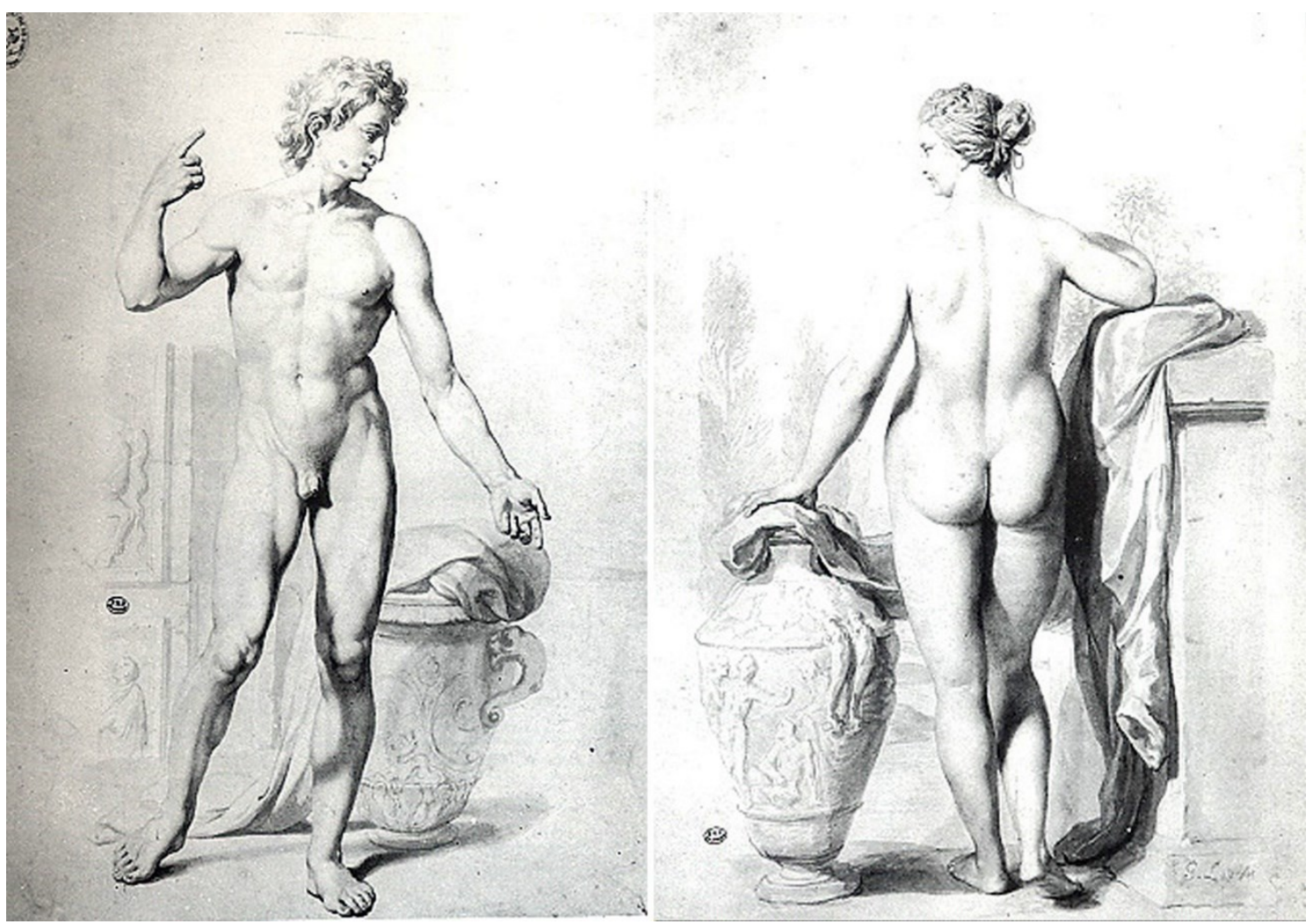

Fig. 4. Gerard de Lairesse, Dibujo de hombre joven y Dibujo de mujer joven, Biblioteca de Módena (inv. $\mathrm{n}^{\circ}$ Ms 26 )

apoyada en un lateral del plinto sobre el que se sienta la alegoría del dibujo, y que Johannes aprovecha para colocar su firma en la pintura del museo de Rouen, ha desaparecido ${ }^{16}$. Otras diferencias entre las dos pinturas incluyen el cambio en el paisaje, optando por una hilera de árboles en perspectiva hacia el horizonte en la obra de Rouen, frente al sentido más de bosque en la obra aquí tratada. De este modo, se logra una visión más uniforme del espacio en este lado, sin distraer al espectador por esa naturaleza organizada que presenta el ejemplar de Rouen. A pesar de estos cambios, la composición no ha variado, aunque las dimensiones del lienzo de Rouen son mayores, 95 $x 76,2 \mathrm{~cm}$. , que las de la obra de la colección Epiarte. La pintura del museo francés se fecha entre 1710 y 1715 , cronología que puede aplicarse también a la réplica que estudiamos.

En esta composición es evidente que Johannes de Lairesse sigue el enigmático y complejo universo de su padre. Los modelos de la Razón y del aprendiz se encuentran, con igual anatomía y rostro, en dibujos firmados "De Lairesse" y fechados hacia 1685, en la Biblioteca Universitaria de Módena (inv. MS96) ${ }^{17}$. (Fig. 4) Especialmente interesantes en este juego de dependencias son los grabados de Cabezas femeninas de Gérard de Lairesse (aguafuerte de 1670), utilizadas para la alegoría del Dibujo que protagoniza el tema aquí estudiado ${ }^{18}$. (Fig. 5)

\footnotetext{
${ }^{16}$ Bakhuÿs et al., Tableaux flamands, p.209.; De Jongh, Artist's Apprentice, p. 207.

17 Véase Roy, Gérard de Lairesse, p. 395, D 55 y D 53.

18 Holstein, 1955, no 97.
} 


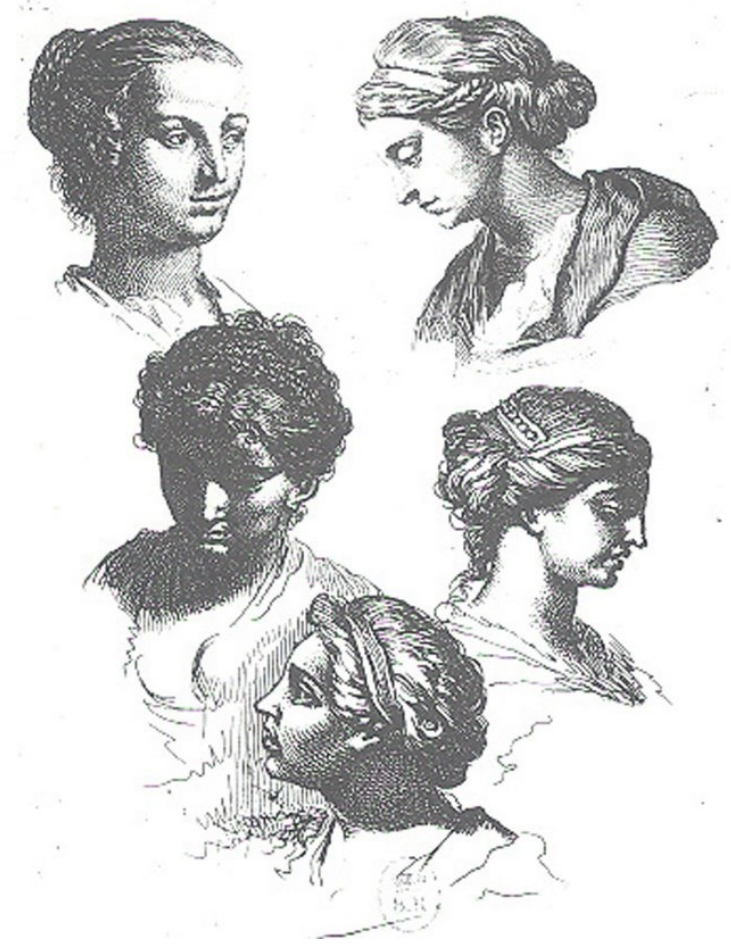

Fig. 5. Gérard de Lairesse, Estudio de cabezas, grabado.

Sería reiterativo señalar las numerosas composiciones de Gérard de Lairesse que coinciden con las de su hijo Johannes. Ambo repiten ese esquema horizontal donde la vertical la marca la columna divisoria. Sirva de ejemplo El banquete de Cleopatra del Rijksmuseum de Ámsterdam ${ }^{19}$, (Fig. 6) con la reina de Egipto recostada de manera similar a Minerva en la pintura aquí estudiada. En ésta, Johannes de Lairesse logra una delicada unidad tonal, un perfecto dominio del dibujo y la seducción del espíritu grecorromano, adoptado del estilo de su padre. Estas escenas son premonitorias de la estética que Johann Joachim Winckelmann (1717-1768) defenderá con el Neoclasicismo, donde la teatralidad y la puesta en escena entronca con la distinción de los esquemas clásicos. Este tipo de pintura de los Lairesse tendrá gran éxito entre la élite de fines del siglo XVII y principios del XVIII ${ }^{20}$. Donde la arquitectura y la escultura imponen un claro dominio en estas composiciones. La línea supedita al modelado y al color, que se doblegan a la perfección y a la belleza de las formas. Una estética y una concepción pictórica que el joven Johannes sigue de los predicamentos de su padre: "Un joven artista no debe trabajar sin los ejemplos de las Estatuas de Perrier, la Iconología de Cesare Ripa, el Poder romano de Oudaen, y otros libros de antigüedades"21.

\footnotetext{
${ }^{19}$ Inv. A. 2115: Roy, Gérard de Lairesse, pp. 270-272.

${ }^{20}$ Roy, Gérard de Lairesse, p. 75.

21 Gerard de Lairesse, The Art of Painting in all its Branches, ed. John Frederick Fritsh, (London:

S'Vandernbergh, 1778), libro X, p. 400.
} 


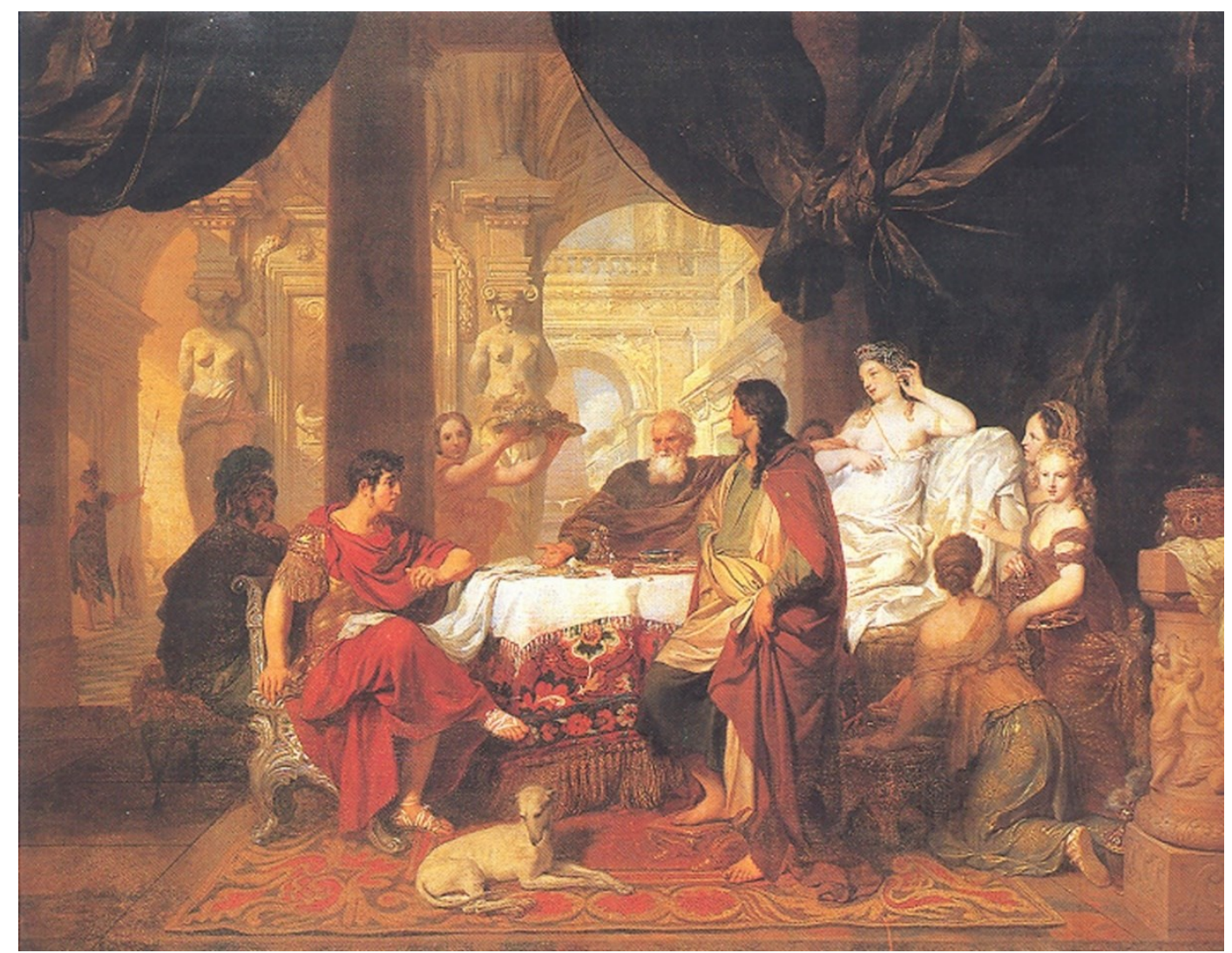

Fig. 6. Gérard de Lairesse, El banquete de Cleopatra, Rijksmusum de Ámsterdam (inv. no A. 2115) 
Bibliografía

Bakhuÿs, Hillegers, Tainturier, y Jonckheers 2009: Diederik Bakhuÿs, Jasper Hillegers, Cécile Tainturier y Koenraad Jonckheers, Tableaux flamands et hollandais du Musée des Beaux-Arts de Rouen, (Paris: Fondation Custodia, 2009).

Blankert 1967: Albert Blankert "Heraclitus en Democritus, in het bijzonder in de Nederlandse kunst van de 17de eeuw", Nederlands Kunsthistorich Jaarboek, vol. 18, pp. 31-124, fig. 49.

Blankert 1999: Albert Blankert et al., Hollands Classicisme in de zeventiendeeeuwse schilderkusnt, Cat. Exp., (Rotterdam-Frankfurt am Main, 1999), pp. 338-340.

Bruselas 2018-2019: Exp., Theoodor van Loon. A Caravaggist painter between Rome and Brussels, BOZAR, Bruselas, 2018-2019.

Bruyn 1988: Jean-Pierre de Bruyn, Erasmus II Quellinus (1607-1678) De Schilderijen met Catalogue Raisonné. Vlaamse Schilders uit de Tijd Van de Grote Meesters, (Freren: Luca, 1988).

Conti 1567: Natale Conti, Mythologiae, sive explicationis fabularum. Libri Decem, (Paris: Thomas Blasius, 1605).

Cornil: Th. Cornil, "Théodore van Loon et la peinture italienne", Bulletin de I'Institut historique belge de Rome, 17, (1936), pp. 187-211.

Hollstein 1955: Friedrich Wilhem Hollstein, Holstein's Dutch and Flemish etchings, engravings, and woodcuts (ca. 1450-1700), T. X, (Amsterdam: M. Hertzberger,1949-2010).

Jongh 1983: Eddy de Jongh, "The Artist's Apprentice and Minerva's Secret: An Allegory of Drawing by Jan de Lairesse", Simiolus: Netherlands Quarterly for the History of Art, 13, no 3/4, (1983), pp. 201 - 217.

Lairesse 1778: Gerard de Lairesse, The Art of Painting in all its Branches, ed. John Frederick Fritsh, (London: S'Vandernbergh, 1778).

Ripa 1644: Cesare Ripa, Iconologia of Uytbeeldingen des Verstands, (Amsterdam: Dirck Pietersz Pers, 1644).

Roy 1992: Alain Roy, Gérard de Lairesse 1640-1711, (Paris: Arthena, 1992). Wurzbach 1910: Alfred von Wurzbach, Niederländisches Künstler-Lexikon, II, (Wien-Leipzig: Halm und Goldmann, 1910).

Recibido: 20/11/2018

Aceptado: 29/11/2018 